FORMATION Formation emploi

Revue française de sciences sociales

115 | Juillet-Septembre 2011

Le développement de l'aide à la personne : quelle professionnalisation?

\title{
Devenir une professionnelle de l'aide à la personne : entre technique, relations et organisation
}

Becoming a home care professional: technical, relational and organisational aspects

Fachfrau in der Lebenshilfe werden: zwischen Technik, Beziehungen und Organisation

Convertirse en una profesional de la ayuda a las personas : entre técnica, relaciones y organización

\section{Gérald Houdeville et Laurent Riot}

\section{OpenEdition}

Journals

Édition électronique

URL : http://journals.openedition.org/formationemploi/3415

ISSN : 2107-0946

Éditeur

La Documentation française

Édition imprimée

Date de publication : 23 septembre 2011

Pagination : 25-36

ISSN : 0759-6340

Référence électronique

Gérald Houdeville et Laurent Riot, « Devenir une professionnelle de l'aide à la personne : entre technique, relations et organisation », Formation emploi [En ligne], 115 | Juillet-Septembre 2011, mis en ligne le 24 septembre 2013, consulté le 30 octobre 2020. URL : http://journals.openedition.org/ formationemploi/3415 


\section{DOSSIER}

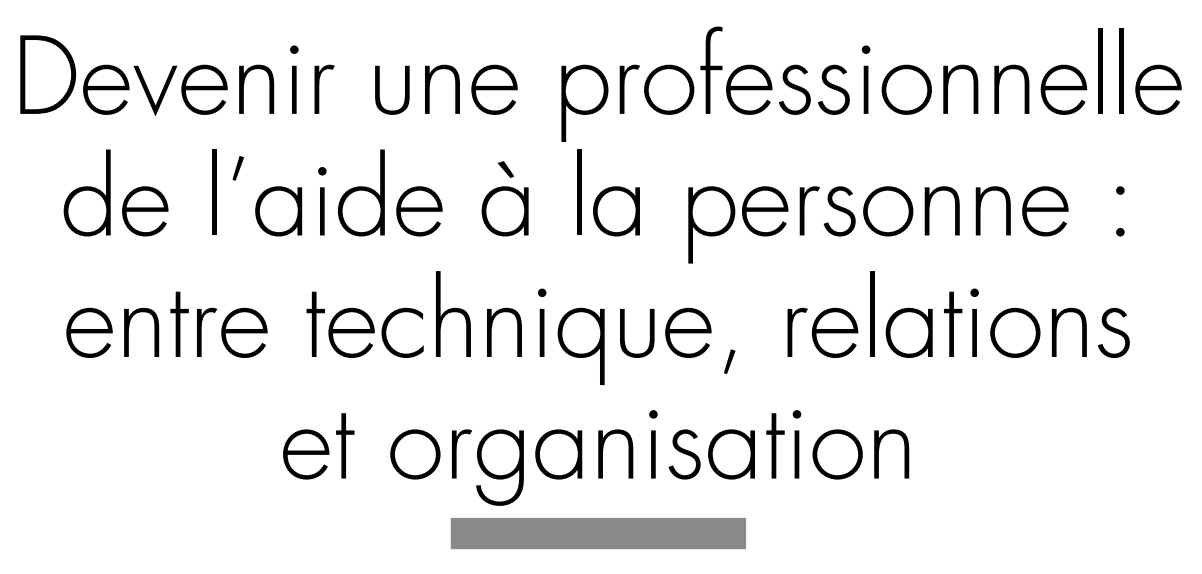

Gérald Houdeville et Laurent Riot ${ }^{*}$

Les formations dans le secteur de l'aide à la personne comportent à la fois une dimension technique et morale, notamment afin d'établir une distance professionnelle avec les usagers.

L'usage actuel de la notion de professionnalisation associé au secteur de l'aide à la personne résulte, pour une grande part, d'une action volontariste de l'État. Ce dernier, à travers ce credo, tente en effet d'élaborer, depuis le début des années 2000, une politique ambitieuse d'emploi et de qualification en direction des femmes, notamment celles en reprise d'activité, demandeuses d'emploi, peu qualifiées. La branche de l'aide à domicile constitue un autre protagoniste incontournable de ce mouvement. Elle est parvenue à imposer un développement conséquent de formations professionnelles de niveau $\mathrm{V}^{1}$ dans ce secteur jusque-là réputé « sans qualification ». Ainsi, les trois quarts des formations examinées dans cet article n'existaient pas il y a encore dix ans. De plus,

\footnotetext{
${ }^{1}$ Selon la nomenclature des niveaux de formation qui définit les niveaux de formation en termes de durée. Le niveau $\mathrm{V}$ correspond à un niveau de formation équivalent à celui du brevet d'études professionnelles (BEP - soit deux ans de scolarité au-delà du premier cycle de l'enseignement du second degré) et du certificat d'aptitude professionnelle (CAP).
}

le seul certificat professionnel qui existait à cette époque, le CAFAD (Certificat d'aptitude aux fonctions d'aide à domicile), ne s'ouvrait qu'aux salariées $\mathrm{du}$ secteur, là où les formations actuelles s'adressent à l'ensemble de la population. Enfin, les Régions

Gérald Houdeville est enseignant-chercheur en sociologie à I'IPSA (Institut de psychologie et sociologie appliquées)/UCO (Université catholique de l'Ouest) à Angers et au CENS (Centre nantais de sociologie) de l'université de Nantes. II a notamment publié : "Une position difficile à maintenir. Entretien avec François Lautier, sociologue en "archi" (1967-2008) », Espaces et sociétés, n 142, juin 2010, pp. 33-48. "De la vocation à l'emploi. La fabrique des dispositions à l'exercice du métier de sociologue après 1945 », Regards sociologiques, $n^{\circ} 36,2009$, pp. 53-68 
Laurent Riot est sociologue au GERS /Groupe d'études et de recherches sociales), chercheur associé au CENS (Centre nantais de sociologie). Il a publié notamment : "Les pratiques des formateurs dans le cadre de dispositifs d'insertion : le projet en théorie, les compromis en pratique ", in de Lescure $E$. et Frétigné $C$., Les métiers de la formation: approches sociologiques, Rennes, PUR, 2010, pp. 157-168. "Entre l'école et l'entreprise : les Missions Locales ", Les Temps modernes, mars-juin 2006, n 637-638-639, pp. 420-443.

sont aujourd'hui un acteur politique important de ce mouvement de professionnalisation. En effet, depuis la loi de décentralisation de 2004, elles financent les formations du travail social et organisent un schéma d'ensemble des formations professionnelles de l'aide à la personne.

De façon consensuelle, ces acteurs considèrent que la professionnalisation de l'aide à la personne doit servir une aide de meilleure qualité à des populations fragilisées ou vulnérables (les personnes âgées dites dépendantes et les handicapés notamment). En effet, cette aide est censée ne plus relever que de la seule bonne volonté des intervenantes à domicile. Elle doit mobiliser des qualités techniques et relationnelles que les nouvelles certifications et diplômes de ce secteur reconnaissent au terme de formations spécifiques. En somme, ces nouvelles certifications doivent renforcer la légitimité professionnelle des intervenantes en poste et

\section{Encadré 1}

\section{Une enquête et ses méthodes}

L'enquête de terrain a été réalisée dans le cadre d'une recherche du Centre régional associé au Céreq des Pays de la Loire et de différents partenaires régionaux intéressés par le développement des formations de l'aide à la personne. Elle a été conduite conjointement à une autre, réalisée auprès d'élèves inscrits dans des filières scolaires sanitaires et sociales. Elle a reçu le soutien financier de la région des Pays de la Loire, Nantes Métropole, Uniformation, la Direction régionale des Affaires sanitaires et sociales, le conseil général de Loire-Atlantique, l'université de Nantes et la Maison de l'emploi d'Angers. Quarante-deux entretiens approfondis ont été réalisés entre septembre 2008 et mars 2009 auprès de femmes en cours de formation - elles sont approximativement un millier à être inscrites dans les formations étudiées. Ces entretiens ont été accompagnés d'observations dans les centres de formation où ces femmes ont été rencontrées. Les situations d'entretien ont constitué, pour certaines, l'opportunité d'évoquer leurs « motivations » (aspect que leurs formateurs leur demandent généralement de travailler). Pour d'autres, cet exercice était parfois l'occasion de manquer un cours. Pour la plupart, l'interview pour parler de soi, à laquelle les formateurs les conviaient à participer, était une expérience peu habituelle $\left(^{*}\right)$. II s'agissait pour nous d'abord de cerner les logiques sociales expliquant le rapport de ces femmes à ces formations nouvellement apparues; ensuite, de définir et de rendre compte des bénéfices qu'elles retirent de la formation en replaçant ces acquis dans le système d'acteurs institutionnels de la formation (formateurs, tuteurs et autres intervenants extérieurs au cours de celle-ci). Ces échanges avec les stagiaires se sont élargis aux formateurs et souvent aux directions d'établissements sollicités dans le cadre de l'enquête. II s'agissait de mieux définir l'organisation sociale de ces formations (une dizaine d'entretiens).

Parallèlement, un questionnaire d'enquête a été conçu afin d'étoffer les premières analyses qualitatives dégagées par l'étude. Ce questionnaire reprend de façon synthétique la grille de questions utilisée lors des interviews. Il a permis d'élargir l'éventail du terrain d'enquête initial [N=232].

$\left.{ }^{*}\right)$ : Par ces précisions, nous entendons rejoindre les approches pour lesquelles l'explicitation de la démarche et le retour sur les conditions de production du matériau empirique, même bref comme ici, sont indispensables au moment d'évaluer la scientificité du travail sociologique. 
des demandeuses d'emploi dirigées vers ce secteur par les services publics de l'emploi. Les quatre formations que nous avons étudiées participent de ce processus : le DEAVS (Diplôme d'État d'auxiliaire de vie sociale) du ministère des Affaires sociales - remplaçant l'ancien CAFAD; le titre ADVF (Assistante de vie aux familles) délivré par le ministère de l'Emploi, ainsi que les CQP EP et $\mathrm{AV}$ (Certificats de qualification professionnelle d'employé polyvalent et d'assistante de vie) attribués par l'IFEP (Institut de formation de la fédération des particuliers employeurs ${ }^{2}$ ).

La professionnalisation n'est pas un leitmotiv propre au seul secteur de l'aide à la personne ; son occurrence s'observe, plus largement, dans des domaines peu qualifiés et relationnels pour lesquels l'État joue un rôle de promoteur, comme l'insertion et la formation par exemple (Demailly, 2010)3. Nous cherchons ici à cerner, d'une part, ce que recouvre ce discours, pour les personnes inscrites dans des formations professionnelles de l'aide à la personne; d'autre part, comment ces formations contribuent à définir les contours d'aptitudes professionnelles nouvelles. Pour mettre à jour ce processus en cours, nous rappellerons d'abord sur quel socle la professionnalisation prend appui dans le cadre de l'étude : quelles sont les caractéristiques des femmes qui se dirigent vers ces formations ? Quels sont les principes qui président à la définition des référentiels des formations professionnelles étudiées? Nous analyserons ensuite comment se traduit l'apprentissage professionnel lors de la formation, alors même qu'aucun acte technique ne semble clairement régir la légitimité professionnelle de l'aide à la personne : cette analyse porte, d'une part, sur la transmission de connaissances et d'une attitude spécifique permettant d'établir une « distance professionnelle » avec les usagers; d'autre part, sur une dialectique active impliquant les formateurs, des tuteurs et d'autres intervenants confrontant ces éléments théoriques à la pratique ordinaire du futur métier.

\footnotetext{
2 Fédération représentant le secteur de l'emploi direct (auparavant désigné « de gré à gré »).

${ }^{3}$ Ce phénomène relève, pour certains domaines, davantage d'une quasi-professionnalisation que d'une structuration sur le modèle des professions établies. À ce titre, les métiers de la formation et de l'insertion, pour lesquels les qualités relationnelles sont mises en avant, présentent de fortes similitudes avec l'aide aux personnes.
}

\section{DES PUBLICS FÉMININS PEU QUALIFIÉS}

Les publics des formations professionnelles considérées sont, sans surprise, essentiellement féminins : dans leur étude sur la promotion des femmes dans l'aide à domicile, G. Doniol-Shaw, E. Lada et $\mathrm{A}$. Dussuet y estiment à $98 \%$ la proportion de femmes (Doniol-Shaw et al., 2007). Nous constatons une prédominance similaire dans notre échantillon (seuls deux stagiaires hommes rencontrés en formation ADVF) ${ }^{4}$. Comme le soulignent différents auteurs (Amossé, 2001 ; Avril, 2006), ces femmes sont, pour une grande part, peu dotées en qualification - plus de $60 \%$ de celles interrogées par questionnaire ne sont pas ou sont peu diplômées (niveaux V et VI de formation $^{5}$ ). Leur trajectoire professionnelle se caractérise par un rapport discontinu à l'emploi. Ces femmes " peu qualifiées » sont par exemple d'anciennes ouvrières de l'industrie textile, auparavant employées à des travaux saisonniers, ou d'anciennes employées de bureau déclassées par l'informatisation. Pour parler comme Christelle Avril (2006), elles trouvent, dans l'aide à la personne, un « refuge » où la main d'œuvre masculine n'exerce pas une concurrence sérieuse. Ces tendances font dire que le secteur reflète aujourd'hui «les visages de la précarité » (Doniol-Shaw et al., 2007).

Pour autant, la population que nous avons rencontrée témoigne de trajectoires relativement diversifiées. C'est peut-être là un effet de l'élargissement du recrutement de la main-d'œuvre dans ce secteur, suite aux campagnes publiques de communication récentes. Certes, les stagiaires interrogées sont plutôt issues de milieux modestes et ont souvent rencontré des difficultés sur le marché de l'emploi. Néanmoins, une petite dizaine d'entre elles occupait des postes d'encadrement moyen avant d'entrer en formation. Par ailleurs, leur inscription en formation - consécutive à la détérioration de leurs conditions de travail ou bien à des évènements familiaux et conjugaux intervenus brusquement - représente, pour elles,

\footnotetext{
${ }^{4}$ Cette tendance explique l'usage systématique du féminin dans notre texte pour désigner des stagiaires.

5 Le niveau VI correspond à un niveau de formation équivalent à la scolarité obligatoire.
} 
une reconversion délibérée pour rejoindre un secteur qu'elles considèrent en pleine expansion (c'est, du reste, de cette manière qu'il leur est généralement présenté par les orienteurs). Pour une partie d'entre elles, il n'y a quasiment aucune connaissance préalable de l'aide à la personne en tant que secteur d'emploi ; elles proviennent d'un autre secteur d'activité ou entrent sur le marché de l'emploi sans nécessairement connaître, dans leur parcours, une expérience bénévole ou salariée d'aide. Pour d'autres, qui ont déjà un pied dans ce secteur en tant que bénévole ou salariée, l'entrée en formation correspond à une quête d'évolution personnelle et professionnelle, voire une recherche de sécurisation de leur emploi. Ces femmes cherchent, à travers la formation, à conforter leur emploi et à faire reconnaître leur métier de façon matérielle et symbolique. Leur position s'inscrit néanmoins dans une organisation professionnelle qui, pour plusieurs raisons, n'est pas toujours favorable au développement de la qualification.

\section{L'« ORGANISATION SOCIALE »6 DES FORMATIONS}

La situation en formation des personnes interviewées dépend de tout un système d'acteurs : organismes de formation conventionnés ${ }^{7}$, administrations d'État délivrant des certifications professionnelles reconnues par la branche d'activité (diplômes d'État, certifications et qualifications professionnelles), financeurs (notamment les Régions depuis 2004) et, enfin, professionnels du secteur de l'aide à domicile - principalement des administrateurs et des personnels d'organismes employeurs (associations, municipalités et entreprises). Bien qu'ils ne partagent pas tous les mêmes intérêts, ces acteurs ont en commun la volonté de faire reconnaître des aptitudes professionnelles nouvelles. Le contrôle des certifications attestant de ces aptitudes

\footnotetext{
${ }^{6}$ On emprunte cette formule à Cicourel A. (1968), The social organization of juvenile justice, London, Heinemann educationnal book Ltd.

7 CEFRAS (Centres de formation et de recherche à la relation d'aide et de soins), MFR (maisons familiales rurales), CEAS (Centres d'étude et d'action sociale), AFPA (Association nationale pour la formation professionnelle des adultes), GRETA (Groupements d'établissements publics d'enseignement : ils organisent des actions de formation continue pour les adultes).
}

professionnelles est au cœur de leur mobilisation : à travers une offre de formations qualifiantes, ils cherchent à instaurer une structuration professionnelle du secteur. Les organismes de formation étudiés sont conventionnés non seulement pour leurs compétences reconnues en matière de pédagogie mais aussi pour leurs rapports avec le milieu professionnel et, par-là, pour leur connaissance de celui-ci. Les relations qu'ils affichent avec des professionnels de l'aide à la personne sont un gage de leur crédibilité auprès des certificateurs. Ces relations prennent des formes variées selon les cas et croisent en partie les intérêts des professionnels (participation au conseil d'administration, intervention dans les formations, etc.).

Ces liens sont également intéressants pour les professionnels impliqués dans le système d'acteurs ; ils peuvent en effet accueillir un certain nombre de bénévoles en stage et retirent des bénéfices symboliques au travers de leur participation à des jurys professionnels. Dans ce cadre, le motif de l'appel aux professionnels est particulièrement valorisant. On leur reconnaît un statut d'expert qui ne les réduit pas au seul rôle instrumental de fournisseurs de stages - et/ ou d'employeurs potentiels : en DEAVS, trois stages de cinq à six semaines, auprès de publics diversifiés, sont obligatoires

La conformité des contenus pédagogiques aux textes officiels en vigueur constitue un autre élément important qui fonde la légitimité à agir des organismes de formation. Ces textes définissent l'orientation générale des formations professionnelles de l'aide à la personne au plan national, avec des nuances propres aux institutions certificatrices. Dans les faits, les repères fournis par les référentiels d'emploi et de formation sont suffisamment larges pour que les organismes de formation se les (ré)approprient selon leur histoire propre et leurs expériences pédagogiques. En outre, ces référentiels se révèlent assez proches les uns des autres : ils visent, pour l'essentiel, la transmission de connaissances théoriques plus ou moins développées et de techniques centrées sur l'accompagnement individuel. Deux grands domaines de connaissances y sont identifiables.

Premièrement, l'apprentissage d'un vocabulaire spécifique pour désigner les problèmes des personnes fragiles à accompagner. Ces difficultés sont définies 
par une approche issue à la fois de la psychologie et de l'intervention sanitaire et sociale. On attend ici l'intériorisation de rudiments de psychologie (le développement de l'enfant, la psychologie de la personne âgée et handicapée, etc.) ainsi qu'une connaissance des pathologies les plus répandues au sein de ces populations. Dans le même registre, les élèves doivent apprendre des gestes professionnels requis pour accompagner ces personnes fragilisées. Ces gestes se déclinent en plusieurs sous-catégories selon la proximité corporelle qu'ils induisent vis-àvis des personnes aidées : les actes les plus éloignés du corps sont les techniques de l'entretien ménager et de la préparation culinaire ; les plus proches sont les transferts (pour l'aide au déplacement et à l'habillage) ainsi que l'aide à la toilette ; enfin, entre ces deux pôles figurent des actes intermédiaires, comme l'entretien et le rangement du linge.

Un deuxième registre de connaissances a trait au cadre institutionnel et légal dans lequel les futures intervenantes seront amenées à travailler, en lien avec d'autres institutions et intervenants. La maîtrise de ces connaissances, en fin de formation, place les stagiaires dans une position à la fois d'expertise et de conseil auprès des populations fragilisées et de leur famille. Malgré ces convergences de vues, des différences s'observent entre les trois certifications étudiées quant à l'importance accordée à ces niveaux d'intervention : dans la tradition sanitaire et sociale, le DEAVS, par exemple, privilégie les actes comme les transferts et l'aide à la toilette (proches de ce qu'accomplissent les aides soignantes) ; les deux autres certifications semblent accorder, quant à elles, plus de temps aux techniques ménagères et culinaires. Un maître mot, cependant, qualifie le type d'accompagnement attendu au terme de ces formations : « faire avec » et non « à la place » des personnes aidées.

\section{$L A$ « BONNE » DISTANCE PROFESSIONNELLE}

On aurait tort de concevoir ce cadre général comme une simple enveloppe formelle sans effet sur les stagiaires. Un de ces effets est la reconnaissance, chez celles-ci, du sens spécifique du professionnalisme produit par l'organisation sociale du secteur. La légitimité des intervenantes à domicile ne repose pas sur la maîtrise d'actes techniques bien identifiés - comme peuvent l'être les actes médicaux ou infirmiers ; cependant, elle se fonde, majoritairement, sur une morale ${ }^{8}$ de la distance professionnelle vis-à-vis des personnes fragilisées. «Il faut mettre des barrières dans les relations », « On ne doit pas s'attacher aux personnes », «ne pas emporter chez soi les problèmes des gens", etc. sont autant d'expressions que reprennent à leur compte les personnes interrogées. Elles sont significatives de la leçon de distance que s'efforcent de transmettre les formateurs en insistant sur deux aspects complémentaires : une mise à distance visant d'abord la préservation de l'autonomie des usagers (assister à l'accomplissement d'actes ordinaires essentiels de l'existence sans les accomplir à la place des usagers) et une mise à distance visant la préservation psychologique des intervenantes (éviter l'engagement affectif dans la gestion de relations qui peuvent s'avérer émotionnellement difficiles). Cette morale s'illustre aussi par le suivi de conseils pratiques afin d'éviter des relations familières avec les usagers et, par-là, de faire accepter, par ces derniers, leur statut de professionnelles. Ainsi, « il faut » prévoir d'apporter sa propre bouteille d'eau pour éviter d'avoir à demander à boire aux personnes aidées, s'arranger pour ne pas avoir à emprunter leurs toilettes, etc. Bref, il s'agit de s'afficher comme une présence bienveillante et agissante dans les domaines domestiques et sociaux/psychosociaux, sans pour autant se montrer trop proche ; cette posture implique un véritable «travail émotionnel » (Hochschild, 2033) conforme aux situations de travail de l'aide à la personne.

Les savoirs théoriques et les recommandations pratiques sont orientés de manière à nourrir - gage de la légitimité professionnelle de l'aide - une mise à distance des personnes singulières auxquelles les futures auxiliaires de vie sociale seront confrontées. L'action éducative des formateurs vise fondamentalement à changer dans ce sens le regard de leurs élèves vis-à-vis des personnes auprès desquelles elles

\footnotetext{
${ }^{8}$ On reprend ici un terme de Durkheim qui définit la morale professionnelle comme un ensemble de "règles de conduites sanctionnées [...] qui commandent aux individus, qui les obligent à agir de telle ou telle manière, qui imposent des bornes à leurs penchants et leur défendent d'aller plus loin ». Emile Durkheim, Leçons de sociologie, Paris, PUF, 1950, p. 42 et p. 46.
} 
interviennent dès les périodes de stage. Ils s'efforcent de susciter une vision radicalement différente de la vision profane qui était la leur avant le début de la formation. La transmission des savoirs théoriques participe pleinement de cette distanciation par rapport aux situations concrètes auxquelles les élèves sont confrontées : considérer autrement les personnes fragilisées, ne pas les percevoir exclusivement comme des individus singuliers mais les envisager à travers un certain nombre de caractéristiques objectives dont elles sont porteuses (une classe d'âge, une catégorie de handicap, etc.). L'enjeu de la transmission de ces connaissances est d'amener les élèves à partager, avec d'autres « professionnels », un vocabulaire spécifique. L'exemple suivant témoigne de l'importance de ce changement de regard, acquis, semble-t-il, grâce aux apports théoriques : "Les Parkinson, les Alzheimer, l'arthrose, tout ça, c'est des maladies qu'avant... Avant, j'aurais pas pu donner de définition de ces maladies-là. Avoir des définitions précises, ça aide à voir autrement ces personnes-là, savoir comment on peut s'y prendre avec elles, c'est super important ». (femme, 40 ans, DEAVS)

À côté de la transmission de savoirs théoriques, tout un ensemble de savoir-être, de positionnements pratiques face aux personnes aidées - teinté, le plus souvent, de justifications théoriques et/ou scientifiques - est également inculqué. Il s'agit de façonner, chez l'apprenant, de véritables dispositions relationnelles basées sur l'adoption de typifications ou schémas typificatoires ${ }^{9}$ spécifiques, c'est-à-dire de modèles relationnels permettent d'appréhender et de traiter les personnes fragilisées dans le cadre d'interventions professionnalisées.

\section{IL Y A TECHNIQUE ET TECHNIQUE}

Le pôle relationnel de l'activité future est mis en avant dans la description que les femmes interviewées font de leur apprentissage : il s'agit clairement pour

\footnotetext{
${ }^{9}$ Ce vocabulaire théorique, relatif aux schémas de pensée qui s'imposent, dans la vie sociale, à la définition des diverses relations dans lesquelles sont insérés les individus, est emprunté à Berger P., Luckmann T. (1986), La construction sociale de la réalité, Paris, Méridiens Klincksieck, pp. 46-51.
}

elles de se distinguer de la figure repoussoir de l'aide ménagère qui identifie les tâches qui lui incombent à la seule sphère du ménage - « on n'est pas là pour faire que le ménage », leur a-t-on souvent entendu dire. Elles expriment une détestation assez franche pour "les mémères qui travaillent en chaussons, enfermées dans la routine sans jamais se remettre en question » (femme, 29 ans, DEAVS) ; ou bien pour des manières de travailler qui ne permettent pas de mettre en avant les compétences relationnelles mobilisées dans leur travail : en institution, en maison de retraite notamment, tout apparaît programmé selon une logique du rendement qui ne permet pas d'être à l'écoute des personnes ; c'est, pour elles, l'occasion de se démarquer des personnels qui, à leurs yeux, font leur métier sans beaucoup d'humanité comme des « robots qui vous apprennent bien l'ergonomie, les techniques pour les toilettes, les techniques pour passer du fauteuil au lit, beaucoup de manipulations, mais n'ont pas le temps : pas le temps de parler, pas le temps de discuter d'un problème ou d'un autre » (femme, 42 ans, DEAVS).

Néanmoins, sous l'angle de tout un ensemble de techniques qui s'apparentent aux «techniques du corps » (Mauss, 1950) (apprentissages de techniques de transferts - aide au déplacement de personnes invalides -, de l'aide à la toilette ainsi que de techniques faisant écho à des rudiments d'ergonomie appris en centre de formation), les stages sont également souvent vécus dans le même temps comme des expériences décisives permettant de quitter le statut antérieur de profane :

« Je me suis fait reprendre par ma tutrice qui m'avait dit : "tu feras la chambre à fond". Je n'avais pas nettoyé le rebord extérieur de la fenêtre. Donc elle est passée derrière moi en disant : "tu n'as pas fait le rebord extérieur de la fenêtre, tu n'as pas fait la chambre à fond alors que je t'avais demandé de la faire à fond, tu as oublié le rebord extérieur de la fenêtre”. Bon, c'était pénible, j'étais à peu près sûre que je ne ferai jamais rien de bien avec elle... à la fin de la semaine, elle me dit: "tu ne m'as pas entendue, je t'ai appelé collègue”. Ah bah, non, même pas, parce qu'à force, tu m'envoies tellement de trucs dans la tête que j'entends même plus que tu m'appelles collègue le vendredi. Pour moi, j'avais pas l'impression d'être différente le vendredi du lundi, pourtant, j'avais dî, mais je me maîtrisais, genre, il faut que je sois comme 
ça, il faut que je sois comme ça, je suis rentrée dans un moule, ce n'était plus moi. » (femme, 42 ans, DEAVS)

Les entretiens invitent à nuancer l'analyse du rapport de ces femmes à la technique. Il ne s'agit pas d'un rapport fait uniquement du rejet de cette dimension au profit du pôle relationnel de leur activité, qui serait seul susceptible d'en porter une image valorisée ; la maîtrise des actes techniques fait partie intégrante de la définition professionnelle que ces femmes donnent de leur activité - qu'on leur ait proposé ou bien qu'elles soient parvenues à en négocier l'initiation :

« J'ai eu de la chance parce que j'ai travaillé avec une équipe de jeunes qui sortaient tout juste de l'école d'aide soignante. Au niveau ergonomie, j'ai beaucoup appris, parce qu'ils parlent que de ça, les jeunes qui sortent de l'école d'aides soignantes. Ergonomie, techniques pour les toilettes, transferts [...] En stage [foyer logement], j'ai appris à me servir d'un lèvemalade. J'ai appris aussi, ça je devrais pas le dire, à faire des pansements. C'est un soin infirmier, normalement, mais dans les petits foyers logements comme ça, on est obligé de faire un peu de tout. » (femme, 40 ans, DEAVS)

«J'ai appris plein de trucs, comme par exemple la cuisine, parce que moi qui suis pas une tip top de la cuisine du coup... parce qu'il fallait faire tout sur place, il n'y avait pas de surgelé, enfin tous les plats c'est nous qui les préparions pour une trentaine de résidents [...] j'ai appris beaucoup de choses, il y avait surtout à me remettre dans le bain, les lever, les coucher parce que bon c'est quand même des personnes âgées. » (femme, 24 ans, DEAVS)

Soulignons toutefois que la technique, jugée comme valorisante, et invoquée par les élèves interviewées, renvoie souvent à une catégorie de tâches autre que le ménage, une catégorie proche, en fait, des soins corporels ; dans l'extrait ci-dessous, la femme interrogée oppose explicitement le «balai » qu'elle lâche pour regarder «faire une toilette » :

«Un jour [lors de son stage en maison de retraite], j'ai lâché ma serpillière pour aller voir de plus près les toilettes. J'ai demandé à une collègue "ça te dérange pas si je te regarde en train de faire une toilette?" "Je lui explique que je prépare le DEAVS et tout..." Elle m'a dit : "pas de problème". J'ai lâché mon balai et je l'ai regardée faire une toilette. Au moins, j'ai pu voir une professionnelle faire une toilette. » (femme, 42 ans, DEAVS)

\section{LES STAGES ET LA CONFRONTATION AU REGARD DES PROFESSIONNELLES}

Nous ne donnerions pas une image juste des formations de l'aide à la personne si nous laissions penser que leurs contenus techniques et théoriques se transmettent de façon descendante et mécanique vers les élèves en formation. Ces formations, comme d'autres formations d'adultes, reposent sur une relation complexe liée à leur dynamique propre. Ainsi, un certain nombre de questions et de réflexions pratiques échangées entre différents professionnels et les stagiaires font peu à peu prendre conscience à ces dernières de la réalité et des limites des cadres d'action attendus d'elles.

Ce questionnement ouvre la voie à une sociologie de la pratique en formation. Les stages effectués à l'extérieur des organismes de formation constituent, dans cette perspective, un élément clé pour les stagiaires : à la fois en tant qu'apprentissage « sur le tas » mais aussi comme un ingrédient alimentant l'activité éducative des formateurs.

La plupart des stagiaires disent qu'elles ont d'abord craint de retourner à l'école en rejoignant la formation (témoignant par-là de leur faible disposition à emmagasiner des connaissances en restant «assises à leur table ») ; pour autant, elles présentent les stages comme déclencheurs d'une étape qui a cristallisé un regain d'intérêt lors de la formation. Paradoxalement, cependant, leurs premières impressions sur les stages sont souvent déstabilisantes. Elles sont faites de sentiments mêlés d'attirance et de crainte : attirance pour la connaissance vivante des publics et des gestes du métier (ce que les périodes en centre n'abordent pas) mais crainte vis-à-vis à la fois de la position à tenir auprès des professionnelles contactées sur le terrain et de leur propre regard de professionnelles en devenir. Ainsi, beaucoup de témoignages présentent le premier stage - notamment le stage à domicile - comme une 
expérience troublante, source de questionnements. Elle place les stagiaires dans une position qui rappelle une observation d'Erving Goffman sur les représentations de l'individu en situation d'apprentissage : «L'individu à l'aise dans une situation [professionnelle] laisse supposer qu'il s'est bâti l'expérience nécessaire pour faire face aux menaces et aux chances qui peuvent surgir dans cette situation. [Mais] il a moins appris à connaître le monde qui l'entoure qu'il ne s'est exercé à y faire face » (Goffman, 1973). Seule l'« habileté acquise » permet de considérer les situations professionnelles de façon routinière, alors qu'elles suscitaient auparavant alerte et émotion. Aucun acte technique spécifique ne permet aux stagiaires de tenir un rôle clairement défini devant les usagers ; par ailleurs, les tutrices professionnelles, sur le terrain, semblent tenir le leur des relations nouées avec le public. Dès lors, cette maîtrise de soi chez les stagiaires est source de tensions. L'exemple suivant fournit une illustration de ces problèmes de positionnement, fréquents lors des premiers stages :

« Je suis allée en stage avec cette fameuse tutrice très sévère [...] J'ai fait une semaine avec elle. Elle trouvait que j'étais pas assez impliquée. Moi j'avais beau lui expliquer: "je te connais pas, je connais pas les gens chez qui je vais, j'y suis pour huit jours, mais moi j'ai dû mal le lundi à être au taquet. On sait pas chez qui on va, toi tu les connais, ça fait longtemps que tu $y$ vas, je sais pas comment je dois me comporter" ".. (femme, 42 ans, ADVF)

L'apprentissage sur le tas en institution (maison de retraite, centre pour handicapés) est ressenti de manière différente par les stagiaires. Ce type d'expériences est parfois présenté positivement ; à la différence du stage à domicile, la présence de collectifs de travail (d'aides-soignantes, notamment) peut tour à tour rassurer et faciliter certains apprentissages techniques (les transferts et l'aide à la toilette, par exemple). D'un autre côté, cependant, ces stages confortent par défaut l'idéal professionnel de l'aide à la personne : les stagiaires découvrent en effet un univers où la technicité des interventions sert de contre-modèle aux objectifs relationnels mis en avant lors de leur formation. L'entrée en action des intervenants professionnels (infirmiers, aides soignantes) y est collective et standardisée ; de même, la prise en charge des personnes fragilisées se substitue à un accompagnement individualisé. Les interviews sont émaillées de propos reflétant tantôt l'indignation contre les pratiques professionnelles observées en institution, tantôt la valorisation de situations auxquelles elles n'auraient pas pu être confrontées à domicile :

« Comme stagiaire en maison de retraite, je me suis surtout positionnée en observatrice. J'ai vu des choses où je bouillais, où j'avais beaucoup de mal à ne pas réagir et à dire quelque chose, mais je ne pouvais pas. Souvent, j'ai vu, ce sont des femmes qui exercent ce métier-là depuis vingt ans, voire plus, et pour qui, je pense, il faudrait un break. » (femme, 47 ans, Titre $\mathrm{EP})$

"Je vais pas dire que c'est l'usine, malgré que j'aie travaillé en usine avant, mais bon... Tout est programmé un peu au rendement... les filles prennent pas le temps d'écouter les personnes, c'est vraiment un problème. " (femme, 37 ans, $\mathrm{ADVF}$ )

« Dans cette structure, j'ai fait les nuits parce que j'ai demandé, je voulais découvrir la nuit parce qu'à un moment donné, si je trouve un travail et qu'il faut que je travaille la nuit, je suis en stage, c'est le moment de découvrir ; il n'y a pas eu de souci, la nuit, c'est très important aussi parce que l'importance du bon soin pour les résidents, c'est très important parce qu'ils attendent notre passage et ça les réconforte de passer une bonne nuit. » (femme, 47 ans, DEAVS)

«Ce que je trouve plus difficile à domicile, c'est qu'on est seul ... donc je pense que je vais commencer, si je peux, par travailler en institution pour acquérir une expérience avec les personnes, avec un encadrement pour aller seule ensuite... J'ai fait un stage auprès de jeunes handicapés, c'est difficile, je pense qu'il faut acquérir une expérience professionnelle pour aller travailler à leur contact. Peut être qu'elle peut s'acquérir sur place, mais c'est quand même difficile, j'étais bien encadrée. " (femme, 45 ans, DEAVS)

Malgré leur apparente distorsion, ces dimensions sont complémentaires et font des stages en institution une expérience souvent déterminante. Autrement dit, les élèves en formation présentent souvent les stages à domicile et en institution comme des éléments moteurs de la formation, grâce auxquels elles ont eu le sentiment de commencer à apprendre les rudiments de leur 
futur métier. Le cadre institutionnel semble paradoxalement leur apporter plus de repères que les stages à domicile (ces stages en institution sont d'ailleurs aussi l'occasion pour elles de découvrir « en réduction » leur position future dans la hiérarchie paramédicale sous l'autorité des infirmières).

\section{DÉFINIR COLLECTIVEMENT LES CONTOURS DU PROFESSIONNALISME}

Dans le fonctionnement en circuit fermé des groupes de formation, les formateurs s'efforcent de faire des stages effectués à l'extérieur un socle fédérateur pour inculquer des valeurs professionnelles spécifiques. Ils cultivent «l'esprit de camaraderie » qui naît entre les stagiaires adultes (Bizeul, 1988) - et qui semble différencier ces formations d'adultes de l'école, tant redoutée par certaines stagiaires. Ainsi, ils essaient de faire des périodes passées en centre des moments privilégiés d'échanges pour interpréter l'ensemble des événements que vivent les stagiaires à l'extérieur. Les retours de stage sont des moments forts de ces formations. Ils sont combinés à des activités diverses visant à conforter l'unification du groupe. Des activités comme la préparation culinaire, des temps de discussions plus informels en atelier sur la gestion du linge ou de l'espace domestique, par exemple, valorisent tout un ensemble d'arts de faire quotidiens de ces femmes et interviennent dans les objectifs des formations. Les témoignages d'intervenants extérieurs sur des questions soulevées par les cours ou encore des jeux de rôle évoquant des situations observées en stage sont d'autres procédés qui s'inscrivent dans cette logique. Ils visent une acquisition directe et collective des connaissances formelles à partir de l'expérience des stages sous le contrôle des formateurs.

Cette succession répétée des apprentissages en centre de formation et des expériences de stage façonne, petit à petit, chez les stagiaires, une certaine perception des réalités professionnelles du secteur. Cette évolution n'est pas linéaire. Elle repose sur la confrontation des principes théoriques et des expériences vécues sur le terrain. Les stagiaires appliquent généralement avec enthousiasme les règles d'action qui font la spécificité de l'aide à la personne - accompagnement individualisé des personnes fragiles, basé sur l'écoute et l'analyse de leurs problèmes, définition de plans d'action pour les aider - et nourrissent leur apprentissage grâce à la complexité des situations rencontrées sur le terrain.

Les situations auxquelles elles sont confrontées les conduisent à mobiliser avant tout une forte capacité d'adaptation. Il leur faut s'adapter à la variété des ressources qu'elles trouvent au domicile des personnes qu'elles aident - ressources qui se réduisent parfois au strict minimum, vu l'isolement et la marginalité de certaines d'entre elles - et à la diversité de leurs demandes. Plus largement, les situations rencontrées en stage génèrent de nombreuses incertitudes qui sont ensuite largement débattues et, en quelque sorte, «recadrées » en formation.

Quelques éléments paraissent centraux dans ces débats animés par les formateurs. Leur discussion concourt à définir collectivement les contours du professionnalisme émergeant de l'aide à la personne. Ainsi, en réponse aux attentes des usagers, quelles tâches les futures professionnelles sont-elles autorisées à accomplir? Interviennent-elles pour faire le ménage, apporter une aide ou un réconfort moral, jouer à des jeux de société, effectuer des soins, distribuer des médicaments, etc. ${ }^{10}$ ?

"Ce qui se fait et ce qui ne se fait pas, c'est super intéressant. Moi j'ai beaucoup appris avec des gens pas trop rigides, qui acceptent de voir la réalité. On en a beaucoup parlé avec les formatrices. Par exemple, on nous dit "il faut jamais dire la toilette mais l'aide à la toilette". Mais moi, je suis désolée, quand on intervient auprès de personnes... j'ai eu le cas, par exemple, d'une personne atteinte d'une sclérose en plaque, il n'était même pas capable de bouger le petit doigt. Moi, dans ces cas-là, j'appelle plus ça une aide à la toilette. Il ne faut pas dire qu'on fait une toilette, parce qu'on n'est pas aptes à le faire, c'est les aides soignantes. Mais c'est un peu ridicule! Il y a eu une intervention d'aides soignantes durant la formation 10 L'ambivalence et l'incertitude (à domicile aussi bien qu'en
institution) de la définition des tâches de l'intervenant auprès
de personnes fragilisées - est-il là pour faire le ménage ou pour
apporter une aide, un soutien, un réconfort à la personne? - ont été
bien mises en évidence aussi par Caradec V. (1999), «L'aide ména-
gère : entre technique et relationnel, entre logique personnelle et
logique professionnelle », Cahiers de l'UNASSAD, n 1 , pp. 5-23. 
sur ce sujet, et ça, c'était génial. Parce que qu'estce qu'elles disent, les aides soignantes? C'est elles qui sont aptes, normalement, à les faire, les toilettes, hein. Mais elles sont tellement submergées qu'elles disent qu'elles font appel aux associations d'aide à domicile. » (femme, 40 ans, CQP AV)

Pour les stagiaires, les contradictions entre ce que «la théorie » leur enseigne et ce qu'elles observent en stage tiennent lieu d'un apprentissage finalement crucial, celui de la reconnaissance, par d'autres professionnels (formateurs et autres intervenants), des limites de ce qu'elles sont en capacité de faire.

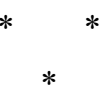

L'objectif était ici de cerner quelques-unes des modalités concrètes de la formation aux métiers de l'aide à la personne dans son déroulement effectif. L'enjeu était de porter un regard sur des dimensions généralement peu visibles d'un secteur sous l'emprise d'un double discours : d'une part, celui d'un secteur considéré comme un gisement d'emplois ; cette vision, taxée de simpliste il y a déjà plus de dix ans (Causse et al., 1997) continue toutefois d'être constitutive d'une politique de développement des services à la personne (Debonneuil, 2007); d'autre part, le discours d'auteurs de conclusions et autres préconisations (Abhervé et Dubois, 2008); ce discours, sous couvert de décrire le processus de professionnalisation en cours, contribue activement, dans les faits, à ce travail de construction à la fois réglementaire, technique et symbolique des métiers de l'aide à la personne comme « profession »- quitte à en dévoiler les difficultés.
On sait peu de choses finalement sur « les registres d'action» (Demailly, 2008) tels qu'ils sont effectivement mis en œuvre dans la formation à ce secteur et déclinés en termes de pratiques. Or, ceux-ci renvoient d'abord à une expérience forte dans un cadre constitué par le jeu des relations entre organismes de formation conventionnés, administrations d'État, financeurs et, enfin, professionnels du secteur de l'aide à domicile : les efforts que tous ces acteurs institutionnels déploient pour transformer la définition sociale des activités d'aide à la personne se traduisent par une action profonde sur les personnes - des femmes, de façon ultra prédominante - qui sont candidates pour s'y former. La professionnalisation en cours est certes à concevoir comme un processus institutionnel ; cependant, elle doit également l'être comme agissant au niveau du sens et de la conscience. Le souci de transmettre la mise à distance des relations interpersonnelles comme disposition d'esprit spécifique à l'égard des personnes fragilisées l'atteste. Ensuite, ces registres d'action reposent autant sur la transmission, par les formateurs, aux femmes en formation, de contenus, de savoirs déterminés - théoriques et pratiques - que sur leur mise à l'épreuve au cours de stages qui révèlent aux stagiaires les écarts entre l'idéal et la réalité. Néanmoins, les formateurs ne proposent pas une vision idéalisée et normative du secteur, en opposition à la prise en charge institutionnelle. Les personnes interviewées déclarent que cette opposition est traitée à part entière en formation et que les discussions qu'elle suscite constituent des ingrédients stimulants pour ajuster sa pratique à la réalité. Ce qu'on doit faire ou ne pas faire par rapport à l'idéal professionnel d'accompagnement apparaît comme le résultat d'une dialectique subtile conduite par les formateurs. 


\section{Bibliographie}

Abhervé M., Dubois P. (2008), « La difficile professionnalisation des emplois dans les services à la personne », Sociologia del lavoro.

Amossé T. (2001), « L’univers des professions (19821999) », Insee Première, $\mathrm{n}^{\circ} 756$.

Avril C. (2006), « Aide à domicile pour personnes âgées : un emploi refuge », in Flahault E. (dir.), L'insertion professionnelle des femmes. Entre contraintes et stratégies d'adaptation, Rennes, PUR, pp. 207-217.

Berger P., Luckmann T. (1986), La construction sociale de la réalité, Paris, Méridiens Klincksieck.

Bizeul D. (1988), « Dix salariés en stage. Une étude d'observation participante », in Le Bouëdec G., Les défis de la formation continue, Paris, L'Harmattan, pp. 135-256.

Caradec V. (1999), « L'aide ménagère : entre technique et relationnel, entre logique personnelle et logique professionnelle ", Cahiers de l'UNASSAD, $\mathrm{n}^{\circ} 1$, pp. 5-23.

Causse L., Fournier C., Labruyère C. (1997), « Le développement des emplois familiaux. Effets sur les métiers de l'aide à domicile », Documents du Cereq, $\mathrm{n}^{\circ} 121$.

CERC (2008), Les services à la personne, Paris, La Documentation française.
Cicourel A. (1968), The Social Organization of Juvenile Justice, Heinenmann Educational Book Ltd, London.

Debonneuil M. (2007), L'espoir économique. Vers la révolution du quaternaire, Paris, Bourin Editeur.

Demailly L. (2008), Politiques de la relation. Approche sociologique des métiers et activités professionnelles relationnelles, Villeneuve d'Asq, Presses Universitaires du Septentrion.

Doniol-Shaw G., Lada E., Dussuet A. (2007), Les parcours professionnels des femmes dans les métiers de l'aide à la personne. Leviers et freins à la qualification et à la promotion, rapport de recherche, Laboratoire Techniques Territoires et Sociétés.

Durkheim E. (1950), Leçons de sociologie, Paris, PUF.

Goffman E. (1973), La mise en scène de la vie quotidienne, Paris, Minuit.

Hochschild A. (2003), « Travail émotionnel, règle de sentiment et structure sociale », Travailler, $\mathrm{n}^{\circ} 9$, pp. 19-49.

Mauss M. (1950), « Les techniques du corps », in Mauss M., Sociologie et anthropologie, Paris, PUF.

Pinaud M. (2004), Le recrutement, la formation et la professionnalisation des salariés du secteur sanitaire et social, avis du Conseil économique et social sur le rapport présenté par Michel Pinaud. 


\title{
Résumé
}

\section{Devenir une professionnelle de l'aide à la personne : entre technique, relations et organisation}

\author{
Gérald Houdeville et Laurent Riot
}

Depuis trente ans, le secteur de l'aide à la personne s'est développé au gré des politiques publiques d'emploi et de lutte contre le chômage féminin. Après avoir encouragé cet essor quantitatif, les pouvoirs publics s'emploient aujourd'hui à améliorer la qualité des prestations des employées de l'aide à la personne : c'est ainsi que la question de leur professionnalisation est récurrente, confortée par une offre de formations qualifiantes de plus en plus étoffée. Sous quelles formes concrètes - dans les centres de formation, sur les lieux de stage - cette professionnalisation se traduitelle ? Comment opère-t-elle ? Quels acteurs implique-t-elle et comment ceux-ci contribuent-ils à la définir ? L'article souligne le double aspect - moral et technique - de ce qui est produit par ces formations en termes de dispositions à agir professionnellement dans ce secteur.

\section{Mots clés}

Service aux particuliers, aide à domicile, professionnalisation, formation professionnelle Journal of Economic Literature : L 84 